\title{
RHIC Pressure Rise and Electron Cloud*
}

\author{
S.Y. Zhang, M. Bai, M. Blaskiewicz, P. Cameron, A. Drees, W. Fischer, D. Gassner, J. Gullotta, \\ P. He, H.C. Hseuh, H. Huang, U. Iriso-Ariz, R. Lee, W.W. MacKay, B. Oerter, V. Ptitsyn, \\ V. Ponnaiyan, T. Roser, T. Satogata, L. Smart, D. Trbojevic, K. Zeno, BNL
}

\section{Abstract}

In RHIC high intensity operation, two types of pressure rise are currently of concern. The first type is at the beam injection, which seems to be caused by the electron multipacting, and the second is the one at the beam transition, where the electron cloud is not the dominant cause. The first type of pressure rise is limiting the beam intensity and the second type might affect the experiments background for very high total beam intensity. In this article, the pressure rises at RHIC are described, and preliminary study results are reported. Some of the unsettled issues and questions are raised, and possible counter measures are discussed.

\section{INTRODUCTION}

In very successful RHIC operations since 2001 [1], two types of pressure rise have shown up, which are of concern for current and future operations, with the increasing beam intensity.

First one is the pressure rise at beam injection, which is very sensitive to bunch intensity and bunch spacing, and tends to approach a saturation for a given beam. Currently, this type of pressure rise is limiting the gold beam bunch intensity of 55-bunch injection at slightly below the design, and it is also preventing the 110-bunch operation with high intensity.

Second type of pressure rise is at beam transition, which is somewhat quasi-exponentially proportional to the total beam intensity, but not sensitive to the bunch intensity and bunch spacing. This pressure rise is not currently limiting the beam intensity. However, in the deuteron-gold beam operation this year, it had caused high background at some of the detectors, and hence hampers further luminisity improvement.

There are several peculiar features of the RHIC pressure rise and electron cloud. For example, bunch spacing in the RHIC is much longer than the ones elsewhere the electron multipacting had been observed. It is suspected that the beam halo scraping at the long straight beam chambers may have produced enough positive ions to help electrons survive the long bunch gap, and hence makes electron multipacting possible. Also higher than usual beam ion gas desorption rate is needed to explain the pressure rise observation, which might be again related to halo grazing angle scraping.

For the counter measures, we have tried and been studying several options, such as baking of the chamber, beam scrubbing, solenoids, and NEG coating.

*Work performed under Contract Number DE-AC02-98CH 10886 with the auspices of the US Department of Energy

\section{PRESSURE RISE AT INJECTION}

In the 2001-2003 RHIC runs, pressure rise at the high intensity beam injection has often occurred in the warm straight sections, which are in total about $1,300 \mathrm{~m}$ in both rings, over the machine circumference of $3,834 \mathrm{~m}$. Usually the pressure rise takes place in part, not all, of the warm sections, with various pressure rise level [2].

This type of pressure rise has limited the gold beam bunch intensity at $9 \times 10^{8}$ ions per bunch for the 55-bunch mode, just below the design of $10^{9}$ ions per bunch. The pressure rise was very sensitive to the bunch spacing, therefore, for the 110-bunch injection, the situation was much worse, and hence this mode was not operational with acceptable bunch intensity. These are illustrated in Fig.1, where the beam intensity and pressure rise are shown for both 55-bunch ( $216 \mathrm{~ns}$ bunch spacing) and 110-bunch (108 ns bunch spacing) mode.

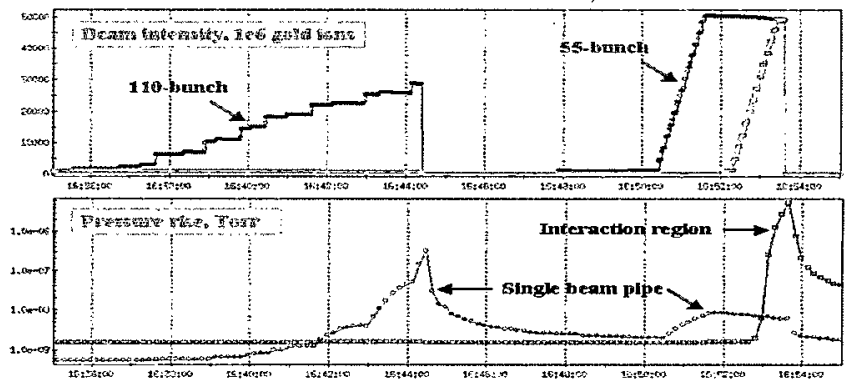

Fig.1. Pressure rise of 55-bunch injection caused valve close in an interaction region. The pressure rise was much higher at a single beam straight for 110-bunch injection.

Longer straights can have lower intensity threshold of the pressure rise. In Fig.2, it is shown that the intensity threshold at single beam straight section, $34 \mathrm{~m}$ long, is a little more than half of that at the interaction region, $20 \mathrm{~m}$.

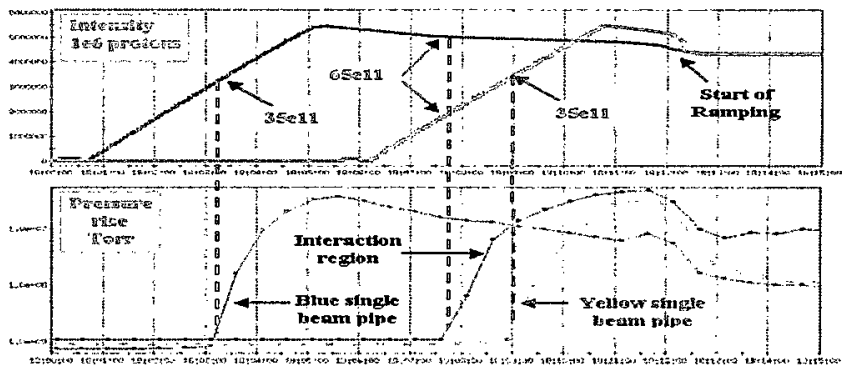

Fig.2. Pressure rise of 55-bunch fill with intensity of $10^{11}$ protons per bunch. Intensity threshold at both blue and yellow straight sections were $35 \times 10^{11}$ protons, and it was $65 \times 10^{11}$ at the interaction straight section. 
Electron cloud seems to be responsible for this type of pressure rise [3-5]. The on-set of the electron multipacting and the strength of the electron signal were in coincidence with the pressure rise. In Fig.3, the peak electron signal with 41 bunches injected into RHIC is shown to compare with the pressure rise. Meanwhile, a solenoid around the electron detector was set off, and on with various strength. The pressure rise is correlated with the electron signal. Also both pressure rise and electron signal were reduced by the solenoid field.

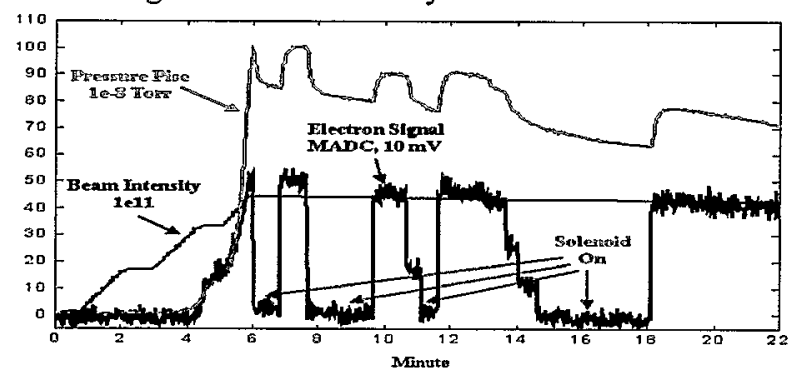

Fig.3. Beam intensity, pressure and the amplitude of electron signal. Solenoid was set off and on, which covers $4 \mathrm{~m}$ in the straight section of $34 \mathrm{~m}$ long.

In Fig.4, the electron signals observed at a scope are shown with the solenoid off, and on at 27 and 5 Gauss.

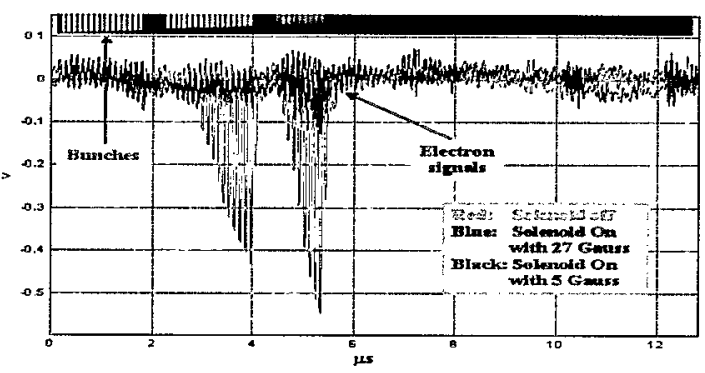

Fig.4. Electron multipacting in a beam injection with gaps, note the gaps re-set the multipacting. The electron signal is reduced when weak solenoid fields applied.

\section{PRESSURE RISE AT TRANSITION}

In the 2003 deuteron-gold (d-Au) run, a new type of pressure rise was observed, which was peaked at the transition, and gradually reduced afterwards. Two features of this pressure rise are of concern. The first is that this pressure rise comprises large mass gases molecule desorption, and the pressure rise takes more than 10 hours to settle down. Therefore, the beam-gas induced detector background might affect experiment data taking. The second feature is that the magnitude of this pressure rise can be approximated as quasiexponentially proportional to the total beam intensity.

In Fig.5, the peak transition pressure rise at 3 of 6 interaction regions, i.e. IR2, IR10 and IR12, of total 74 high intensity ramps in the $\mathrm{d}$-Au run are plotted against the total beam charge intensity in a $10^{9}$ gold ion normalized unit. Data include both 55-bunch and 110bunch ramps, showing that this pressure rise is not sensitive to bunch spacing. Both deuteron and gold beams are accounted in same way, provided the charges are the same, showing that there is no $Z^{2}$ and/or other ion charge effects .

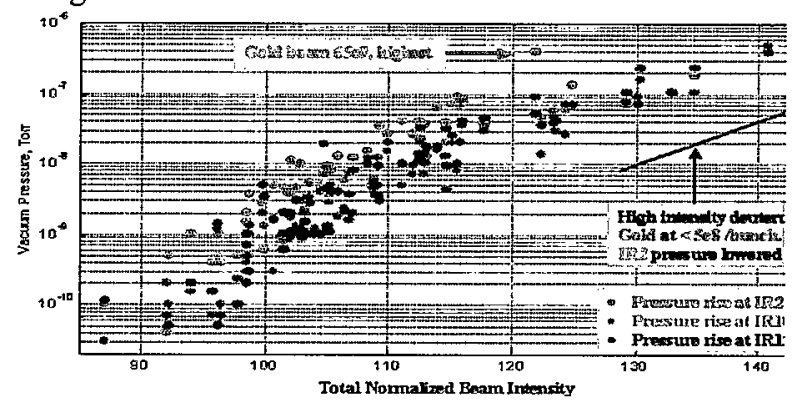

Fig.5. Peak pressure rise of 3 interaction regions at the transition, against total beam intensity. Above total intensity unit of 130, the deuteron beam intensity was higher than gold.

The dominant driving force of this type of pressure rise is probably the beam halo scraping at the chamber wall, with very shallow grazing angle. There is no beam loss correlated with the pressure rise, but at the grazing angle scraping, the gas desorption rate could be very high for high energy ions.

At store, bunches are re-bucketed to the $200 \mathrm{MHz}$ storage RF cavities from the $28 \mathrm{MHz}$ acceleration ones. Re-bucketing shortens bunch length to $5 \mathrm{~ns}$, about the same as that at the transition. Since the beam transverse size is smaller than the one at the transition, the beam potential is higher, yet no pressure rise was observed at the rebucketing. In Fig.6, the pressure rise, the beam potential and beam momentum spread are shown in time. The bunch potential is calculated using $V_{\text {pot }}=I Z_{0} /(2 \pi(0.5+\ln (b / a)))$, where $I$ is the peak current, $Z_{0}=377 \Omega$ is the impedance of free space, $b$ and $a$ are chamber and beam radii, respectively. Bunch intensity of $5 \times 10^{8}$ gold ions is used in calculation. Note that the largest beam momentum spread is at the transition, which may cause some halo scraping.

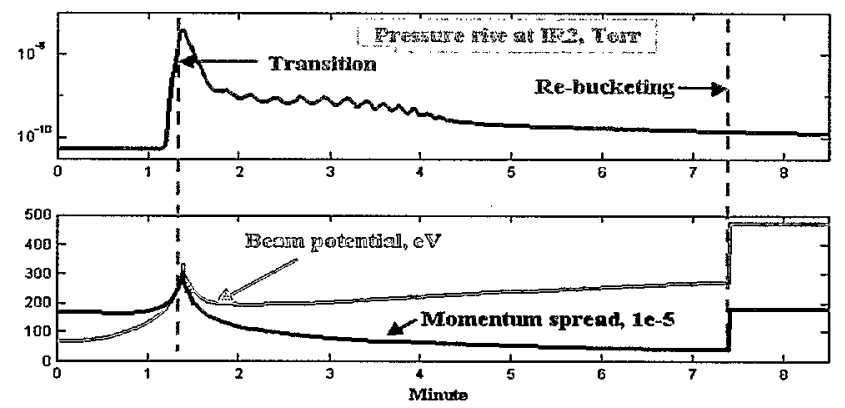

Fig.6. Transition pressure rise at the interaction region IR2. Beam momentum spread is highest at the transition, and the beam potential is highest at the re-bucketing.

Transition pressure rise existed in previous $\mathrm{Au}-\mathrm{Au}$ run, but it was largely ignored, because of the small amplitude. The total intensity then was 80 units, and from Fig.5 it can be seen that below 90 units this effect cannot be identified clearly. The mechanism is not fully understood yet, however, the possibility that this pressure rise becomes a problem in the next Au-Au run cannot be overlooked. 


\section{QUESTIONS AND DISCUSSION}

The RHIC electron multipacting may take place at the bunch spacing as long as 216 ns. As a comparison, with the similar chamber, comparable bunch intensity and shorter bunch, CERN SPS used $50 \mathrm{~ns}$ instead of 25 ns bunch spacing, the electron signal was significantly reduced, and LHC type beam could be injected [6].

Assuming that the beam halo scraping at the long straight beam pipe produces enough positive ions to help the secondary electrons survive the long bunch gap, and hence to make the electron multipacting possible, then several other observations at RHIC might be explained.

- The threshold at the long straight sections is lower than that at shorter sections, as shown in Fig.2.

- A less than $1 \mathrm{~mm}$ radial steering increased pressure rise from $5 \times 10^{-9}$ to $4 \times 10^{-8}$ Torr in some location.

- Smaller transverse beam size at the straight sections improved pressure rise.

- $\quad$ RHIC pressure rise was not distributed evenly in the warm sections. For gold run, it was in fact only taking place in a few locations, and locations may shift.

Experimental data for ion gas desorption rate at the glancing angle scraping was only available recently for low energy ions $[7,8]$. On the other hand, observation in RHIC operations may have already provided some clue. Both in last $\mathrm{Au}-\mathrm{Au}$ run, and the recent d-Au run, beam loss induced pressure rise seems showing that the high energy ion gas desorption rate could be very large. In Fig. 7 , pressure rises of a gold beam fill at two locations are compared with the beam loss monitor data. Assuming that all the lost ions were dissipated at the location $\mathrm{Yi} 2$, the gold ion gas desorption rate is about $1.5 \times 10^{7}$ of $\mathrm{N}_{2}$-equivalent molecules. Note that less then $10 \times 10^{9}$ gold ions lost before the beam dump.

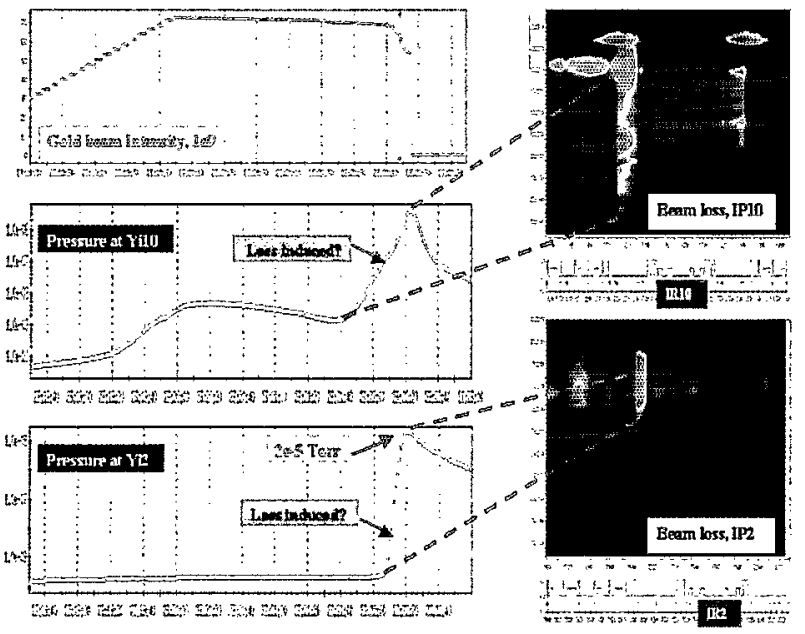

Fig.7. Beam intensity, beam loss induced pressure rises, and the corresponding beam losses at two locations, single beam straight sections YilO and Yi2. Note that the pressure rises at Yi2 by 5 orders of magnitude to $2 \times 10^{-5}$ Torr
This argument may provide a plausible explanation for what happened at the transition pressure rise, for even shallower incident angle. The conclusion is, however, still to be reached. Some studies are necessary to clear this out.

It is possible that electron multipacting took a part in the beam loss induced pressure rise. Because of the positive ions produced by sputtering, the usual space charge force, which confines the electron density, might be reduced, and hence the electron saturation level is raised. However, electron desorption is probably not dominant here. It was observed that strong solenoid field at an interaction region ( housed the STAR detector) was effective for the pressure rise at the injection, but had no effect on the transition pressure rise.

\section{COUNTER MEASURES}

Currently, several counter measures for the pressure rise are undertaken, or under studying at the RHIC.

- Vacuum chamber baking. In last shutdown, additional $40 \%$ of the warm chambers were in-situ baked. In this run, the pressure rise is in general improved in these locations, but not eliminated.

- Solenoid. It has been observed that the solenoid is effective in suppressing the electron multipacting. Since the RHIC pressure rise takes place only in straight sections, solenoid can be applied. However, it will not be effective for the transition pressure rise.

- Beam scrubbing [9]. It has been planned for a study at the RHIC. Since the RHIC pressure rise is very unevenly distributed in the ring, therefore, a complete scrubbing of the ring will be very difficult.

- Beam injection with gaps. It has been shown in a study that beam gaps can be used to reduce the pressure rise. This will be useful in the future operation.

- NEG (Non-evaporable getter) coating [10]. The NEG coating reduces not only the secondary electron yield, but also the electron and ion gas desorption rates. Therefore, it may improve both types of pressure rise at the RHIC, i.e., pressure rise at the injection, and at the transition. NEG coating also provides linear pumping, hence may improve experiment background due to beam-gas. A section test for the NEG coating is under consideration, and potential problems such as aging, activation, etc. are under studying.

\section{REFERENCES}

[1]. T.Roser, these proceedings.

[2]. H.C. Hseuh et al, these proceedings.

[3]. U. Irish-Ariz et al, these proceedings.

[4]. J. Gullotta et al, these proceedings.

[5]. P. He et al, these proceedings.

[6]. J.M. Jimenez, Chamonix XI, CERN, 2001.

[7]. S.Y. Zhang and L.A. Ahrens, PAC1999, p.3294, New York, 1999.

[8]. E. Mahner et al, PRST-AB, Volume 6, 013201, 2003.

[9]. J.M. Jimenez, Mini-Workshop on SPS Scrubbing Run, CERN, 2002.

[10]. C. Benvenuti, PAC 2001, p.602, Chicago, 2001. 